Manuscript for Review

\title{
Low-Power 4-bit Flash Analogue to Digital Converter for Ranging Applications
}

\begin{tabular}{|c|c|}
\hline Journal: & Electronics Letters \\
\hline Manuscript ID: & ELL-2010-2213.R1 \\
\hline Manuscript Type: & Letter \\
\hline $\begin{array}{l}\text { Date Submitted by the } \\
\text { Author: }\end{array}$ & 12-Aug-2010 \\
\hline Complete List of Authors: & $\begin{array}{l}\text { Torfs, Guy; Ghent University, INTEC/IMEC } \\
\text { Li, Zhisheng; Ghent University, INTEC/IMEC } \\
\text { bauwelinck, johan; Ghent University, INTEC/IMEC } \\
\text { Van der Plas, Geert; Imec vzw. } \\
\text { Vandewege, Jan; Ghent University, INTEC/IMEC }\end{array}$ \\
\hline Keywords: & $\begin{array}{l}\text { ANALOGUE-DIGITAL CONVERSION, CMOS INTEGRATED CIRCUITS, } \\
\text { COMPARATORS (CIRCUITS), HIGH-SPEED INTEGRATED CIRCUITS }\end{array}$ \\
\hline
\end{tabular}

\section{SCHOLARONE ${ }^{m}$ Manuscripts}




\section{Low-Power 4-bit Flash Analogue to Digital Converter for Ranging Applications}

G. Torfs, Z. Li, J. Bauwelinck, X. Yin, G. Van der Plas and J. Vandewege

A 4-bit $700 \mathrm{MS} / \mathrm{s}$ Flash ADC is presented in $0.18 \mu \mathrm{m}$ CMOS. By lowering the kickback noise of the individual comparators it was possible to reduce the power consumption to 4.43mW. Improved calibration capabilities resulted in a INL and DNL smaller than 0.25 LSB. These low non linearities give rise to 3.77 effective number of bits at the Nyquist input frequency and this in turn yields an overall figure of merit of $0.46 \mathrm{pJ}$ per conversion step. The lowest figure of merit reported for ADCs with sampling rate above $500 \mathrm{MHz}$ in $0.18 \mu \mathrm{m}$ CMOS.

Introduction: Scaled silicon technologies made it possible to integrate high speed applications in portable devices. A large subsection of these devices are situated into sensor networks. While these sensors are collecting information, which can then be used by the network, used by a central controller or stored in a database, the knowledge of its actual location would be a very important asset. Markets ranging from tracking of postal packets, organization of warehouses to security and healthcare applications can see the advantages of such a technology. Location aware devices such as [1] require a bandwidth of at least $250 \mathrm{MHz}$ to perform an accurate time-of-arrival ranging. The ultrawide band pulse based radio sends a signal with a fast risetime which makes it possible to detect the arrival of the signal within a range of $3 \mathrm{~ns}$, giving a resolution of less than 1m. Flash ADCs are the preferred ADC type for this wideband application requiring 
$500 \mathrm{Msps}$ to digitize an analogue signal with a bandwidth of e.g $125 \mathrm{MHz}$ after IQ downconversion. The required resolution to process the ranging signal is 4-bit, and the focus of this work was to minimize the power consumption, which is one of the most important figures for battery powered sensor nodes. This paper presents such an ADC: a 4-bit, $700 \mathrm{MHz}, 0.46 \mathrm{pJ} /$ conversion-step ADC fabricated in $0.18 \mu \mathrm{m}$ CMOS.

Flash Architecture: For high speed, low resolution ADCs, the flash architecture is a promising approach. [2] presents a $0.8 \mathrm{pJ}$ per conversion 4 bit ADC working at $1 \mathrm{GSps}$ designed in $0.18 \mu \mathrm{m}$ CMOS. An ADC like the one described in [2] offers a low power solution with a linear sample-frequency - power trade-off. The different comparators used in the ADC have a build-in threshold. This is realized by scaling the width of the different input transistors, the resulting offset is then:

$$
\Delta V_{t h}=\frac{V_{G S}-V_{T n}}{2} \frac{\Delta W}{W}
$$

With $\mathrm{V}_{\mathrm{GS}}$ the gate-source voltage of the input transistors and $\mathrm{V}_{\mathrm{Tn}}$ their threshold voltage. This integrated offset reduces the need for a static reference ladder and removes its power consumption. Such a comparator is displayed in Fig 1. A downside of this approach is the kickback noise generated by the comparators. Due to the difference in gate-drain capacitors of transistors $M_{n 2}$ and $M_{n 3}$, a memory effect is introduced. During the reset phase of the comparator (when the sample clock (clk) is going from logic high to logic low), the nodes $X_{L}$ and $X_{R}$ are pulled up to the supply voltage. Assuming the output code was '1' $\left(\mathrm{V}_{\text {out+ }}=\right.$ high, $\mathrm{V}_{\text {out- }}=$ low $)$, node $\mathrm{X}_{\mathrm{R}}$ would initially be charged through $M_{p 6}$ in parallel with the series connection of $M_{n 5}$ and $M_{p 3}$. Node $X_{L}$ will only be charged through transistor $M_{p 1}$. This results in a different time constant for the charging of the two branches, which induces different charges stored on the drain-gate capacitance of $M_{n 2}$ and $M_{n 3}$. Different charges mean different voltages at the input nodes. Depending on the 
driving impedance these can give a big disturbance at the next sample time.

An approach to reduce kickback noise is proposed in [3]. By adding transistors $M_{n 4}$ and $M_{n 5}$ in Fig. 2 the input transistors and their gate capacitance are disconnected from the nodes $X_{L}$ and $X_{R}$ and they are discharged more or less symmetrically during the reset phase via transistor $M_{n 1}$. With the addition of the extra transistors, $M_{n 1}$ can be biased to reduce the current consumption of the comparator which slows down the slewing inside the comparator which will further reduce the kickback noise. The result is an increased sensitivity and giving the possibility to do a more accurate calibration.

Reduction of the kickback noise makes it possible to drive the ADC with a higher impedance, which in turn reduces the power dissipated in this stage. The driver is designed as a $500 \mathrm{MHz}$ two-stage unity gain feedback operational amplifier implemented on the same die providing a high input impedance to the preceding stage, a big advantage compared to [2] which needs a $50 \mathrm{Ohm}$ drive to function properly.

Experimental results and comparison: The ADC has been fabricated in a $0.18 \mu \mathrm{m}$ CMOS technology. The differential input signal was applied to the chip through a balun and the output codes were sampled with a 10GSps digital oscilloscope and processed offline. The linearity was measured by applying a $10 \mathrm{MHz}$ triangle wave. The resulting differential $(\mathrm{DNL})$ and integral non-linearity (INL) is shown in Fig. 3. Without calibration large variations and even missing codes can be observed. Due to process and mismatch errors, the threshold of the comparators can vary up to 2 LSB. After calibration the DNL is below 0.2 LSB and the INL below 0.25 LSB.

The dynamic performance is analysed by applying a full scale differential sine wave. The captured data is then fitted to an ideal sine wave, and with these parameters the signal to noise and distortion ratio (SNDR) and the effective number of bits (ENOB) was 
calculated. The resulting measurements for sample frequencies of 500 and $700 \mathrm{MHz}$ are shown in Fig. 4. The ENOB with a sample frequency of $500 \mathrm{MHz}$ is 3.92 at the Nyquist frequency and 3.77 with a sample clock of $700 \mathrm{MHz}$. After $700 \mathrm{MHz}$ the ENOB starts to degrade quickly due to the speed limitations of the ROM-based digital circuit which converts the thermometer code of the comparators to binary code. Adding a flip-flop in between the comparators and the ROM would solve this timing issue, extending the operating frequency with only a marginal increase of the power consumption.

The measured power consumption is $4.30 \mathrm{~mW}$ at $500 \mathrm{MHz}$ and $5.56 \mathrm{~mW}$ at $700 \mathrm{MHz}$ from a $1.8 \mathrm{~V}$ supply. These figures include the 2 -stage input buffer which consumes $1.13 \mathrm{~mW}$. Without the power consumption of the buffer the figure of merit (FoM) of the ADC core is respectively $0.42 \mathrm{pJ}$ and $0.46 \mathrm{pJ}$ per conversion step at 500 and $700 \mathrm{MHz}$. Including the power consumption of the buffer the FoM is still $0.57 \mathrm{pJ}$ and $0.58 \mathrm{pJ}$ per conversion step respectively. Table 1 compares these figures with the state of the art [2,4-8]. To the author's knowledge, this design achieves the lowest FoM for flash ADCs in this technology node.

Conclusion: A 4 bit $700 \mathrm{MHz}$ ADC in a $0.18 \mu \mathrm{m}$ CMOS technology is designed and tested. It was shown that by reducing the kickback noise of the individual comparators, the power consumption could be lowered to $4.43 \mathrm{~mW}$ without degrading the linearity which at a Nyquist input signal still remained 3.77 effective number of bits, yielding a figure of merit of $0.46 \mathrm{pJ} /$ conversion step. The lowest figure of merit reported for ADCs with sampling rate above $500 \mathrm{MHz}$ in $0.18 \mu \mathrm{m}$ CMOS.

\section{References}

[1] X. Yin, J. Bauwelinck, G. Torfs, P. Demuyter, J. Vandewege, H. Tubbax, J. Wouters, 
P. Debacker, J. Olbrechts, P. Spiessens, F. Stubbe and J. Daneels, "Embedded ranging in ISM band," IET Electronics Letters, Vol. 44, Nr. 17, August 14, 2008, pp. 1043-1045.

[2] P. Nuzzo et al., "A $10.6 \mathrm{~mW} / 0.8 \mathrm{pJ}$ power-scalable $1 \mathrm{GS} / \mathrm{s} 4 \mathrm{~b}$ ADC in $0.18 \mu \mathrm{m}$ CMOS with 5.8 Ghz ERBW," in Proc. 43 ${ }^{\text {rd }}$ Design Automation Conf. (DAC), Jul. 2006, pp. 873-878.

[3] G. Torfs, Z. Li, J. Bauwelinck, X. Yin, J. Vandewege and G. Van der Plas, "A lowpower reduced kick-back comparator with improved calibration for high-speed flash ADCs", IEICE Transactions on Electronics., Vol. E92-C, Nr. 10, October 2009, pp. 1328-1330.

[4] S. Banik, D. Gangopadhyay and T.K. Bhattacharyya, "A Low Power 1.8V 4-bit 400MHz Flash ADC in 0.18 $\mu$ Digital CMOS", Proc. Of VLSID, Jan. 2006, pp 6.

[5] L. Wu, F. Huang, Y. Gao, Y, Wang and J. Cheng, "A 42 mW 2 GS/s 4-bit flash ADC in 0.18- $\mu \mathrm{m}$ CMOS", Int. Conf on WCSP, Nov. 2009, pp 1-5.

[6] A. Ismail, M. Elmasry, "A 6-Bit 1.6-GS/s Low-Power Wideband Flash ADC Converter in 0.13- $\mu \mathrm{m}$ CMOS Technology", IEEE Journal of Solid-State Circuits, Vol. 43, no. 9, Sept. 2008, pp 1982-1990.

[7] C. Sandner, M. Clara, A. Santner, T. Hartig and F. Kuttner, "A 6-bit 1.2-GS/s Low-

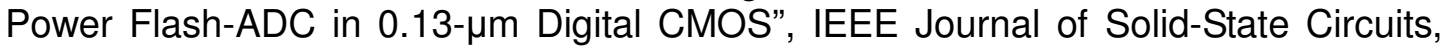
Vol. 40, no. 7, July 2005, pp. 1499-1505.

[8] Y.-Z. Lin, C.-W. Lin and S.-J. Chang, "A 5-bit 3.2GS/s Flash ADC With a Digital Offset Calibration Scheme", IEE Transactions on VLSI Systems, VOL. 18, no. 3, March 2010, pp. 509-513.

\section{Authors' affiliations:}

G. Torfs, Z. Li, J. Bauwelinck, X. Yin and J. Vandewege (Ghent University, INTEC/IMEC, Gent, B-9000, Belgium)

G. Van der Plas (Imec vzw., Leuven, B-3001, Belgium)

E-mail: guy.torfs@intec.UGent.be

\section{Figure captions:}

Fig 1. Comparator with build-in threshold

Fig 2. Reduced kickback comparator

Fig 3. Differential and integral non linearity before calibration and after calibration at a 
sample rate of $700 \mathrm{MHz}$

Fig 4. Effective number of bits versus input frequency at sample rates of $500 \mathrm{MHz}$ and $700 \mathrm{MHz}$

\section{Table captions:}

Table 1. Comparison with state-of-the-art Analog to digital converters. 
Fig 1.

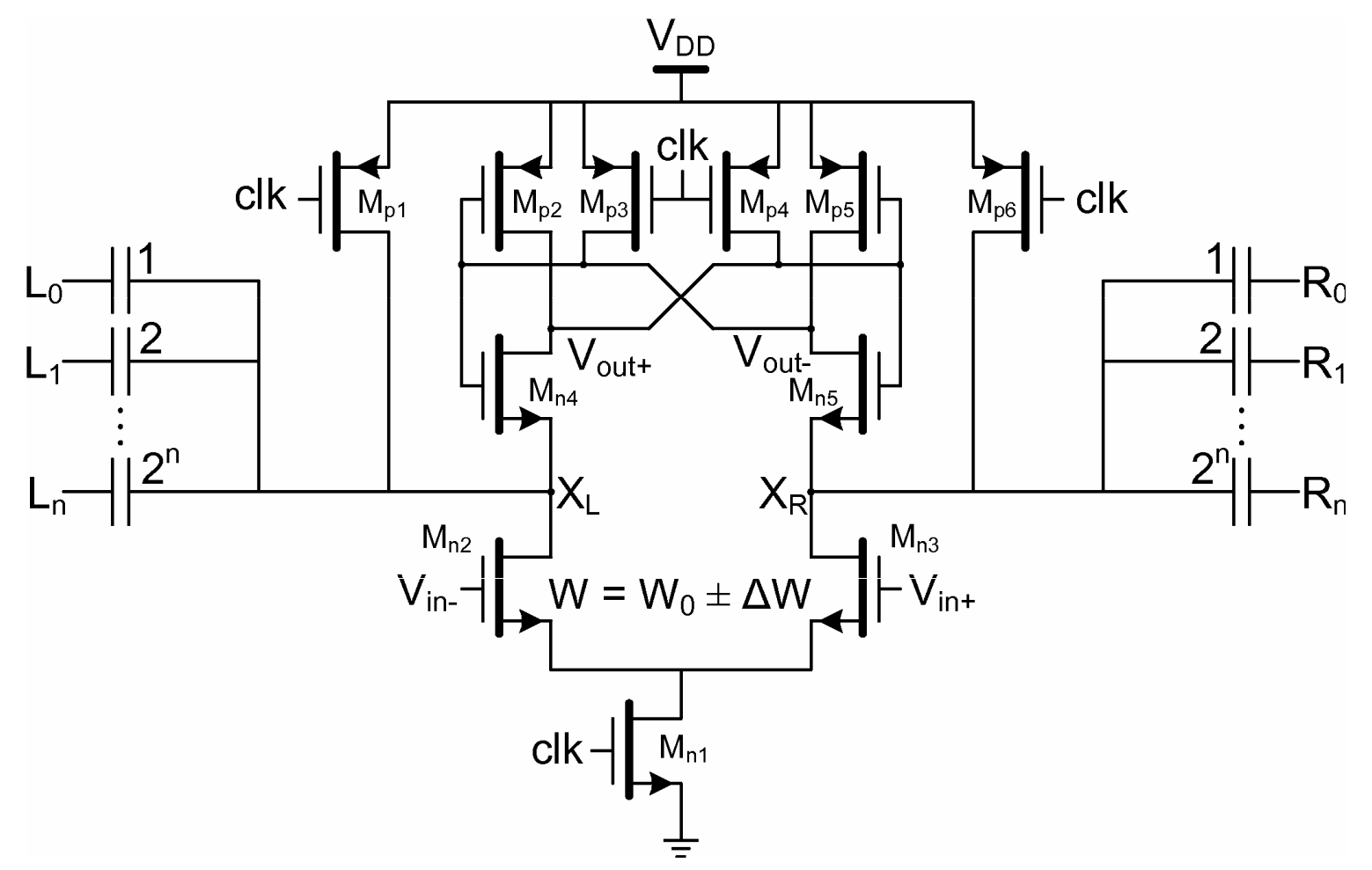


Fig 2.

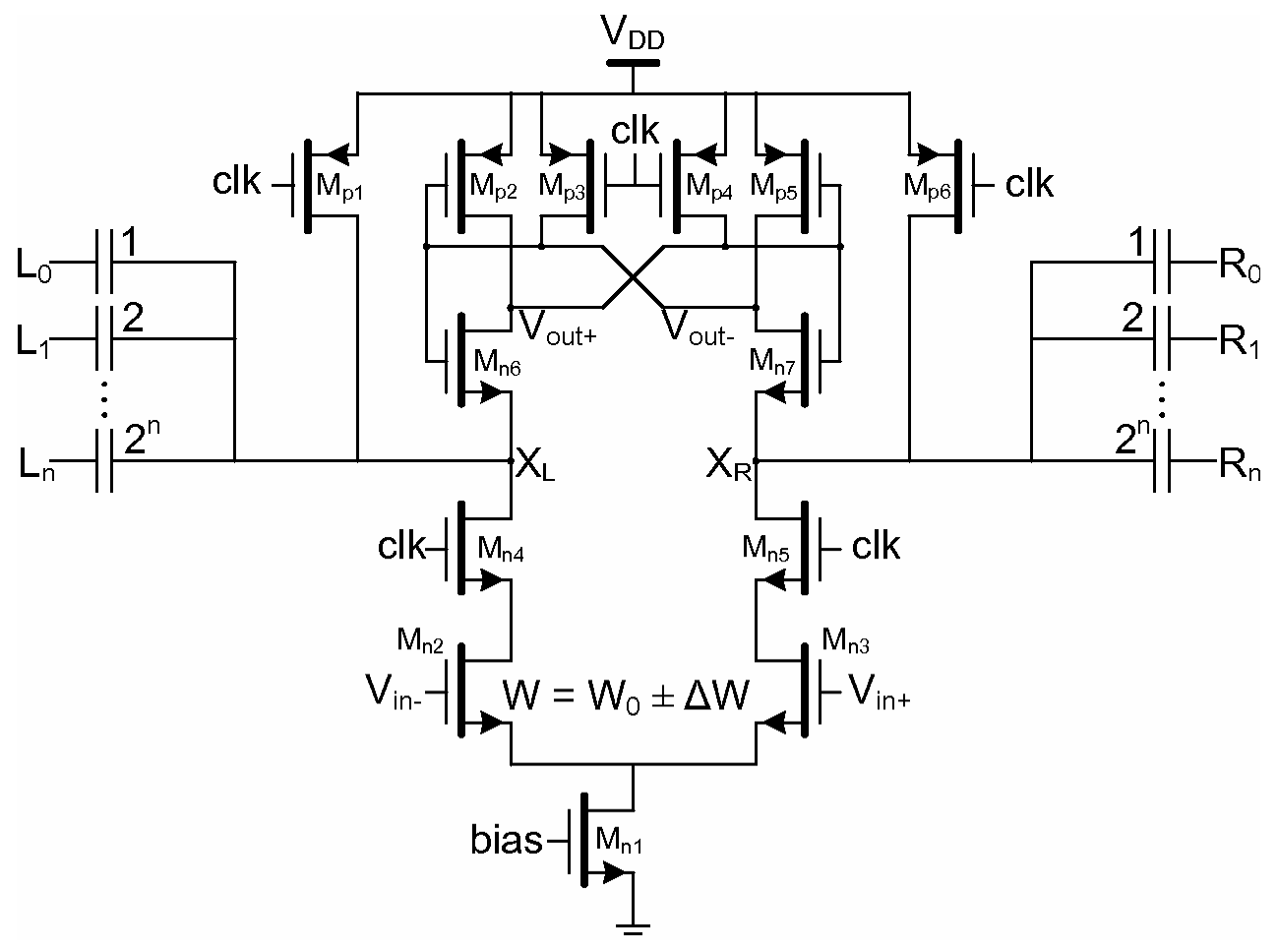


Fig 3.
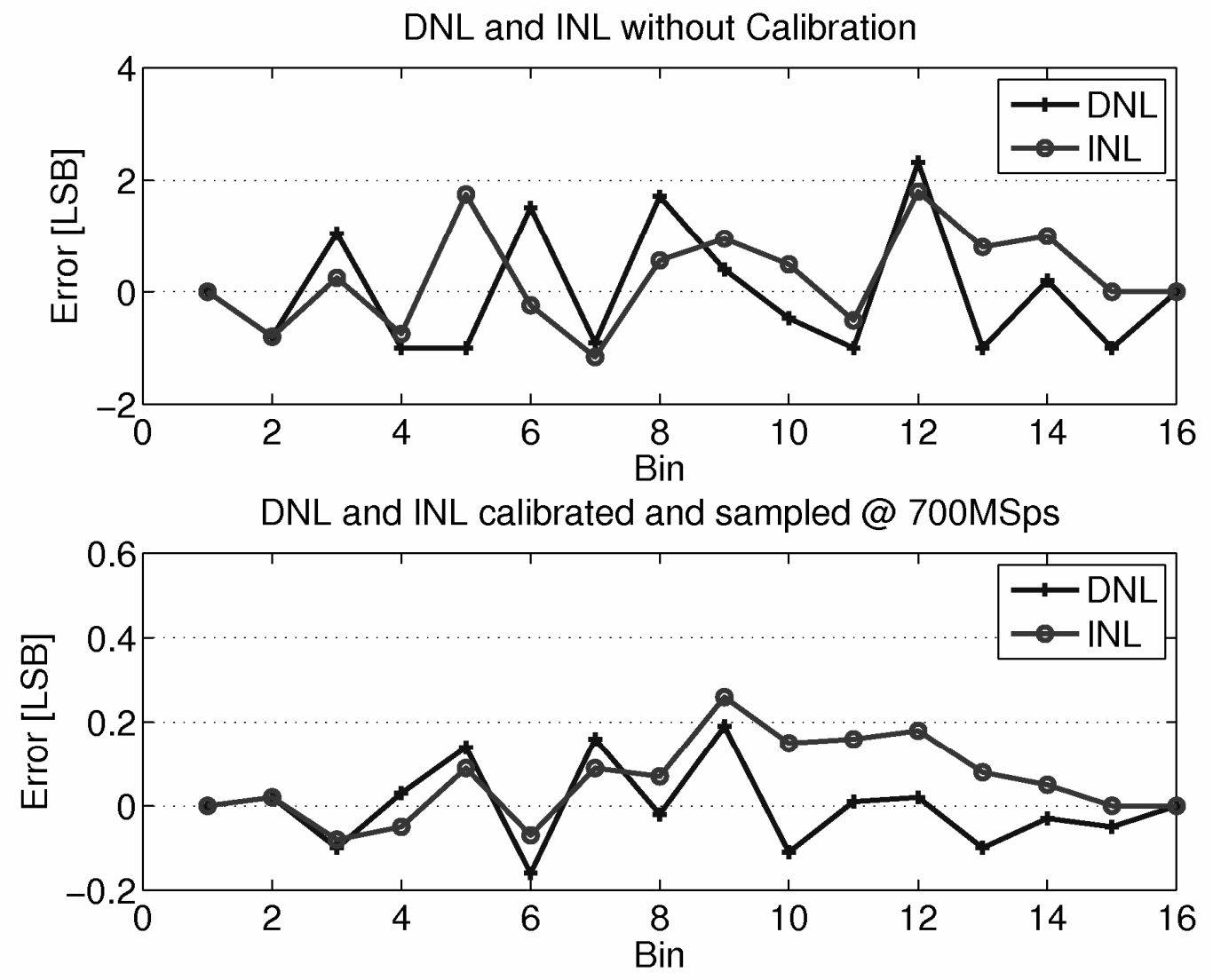
Fig4.

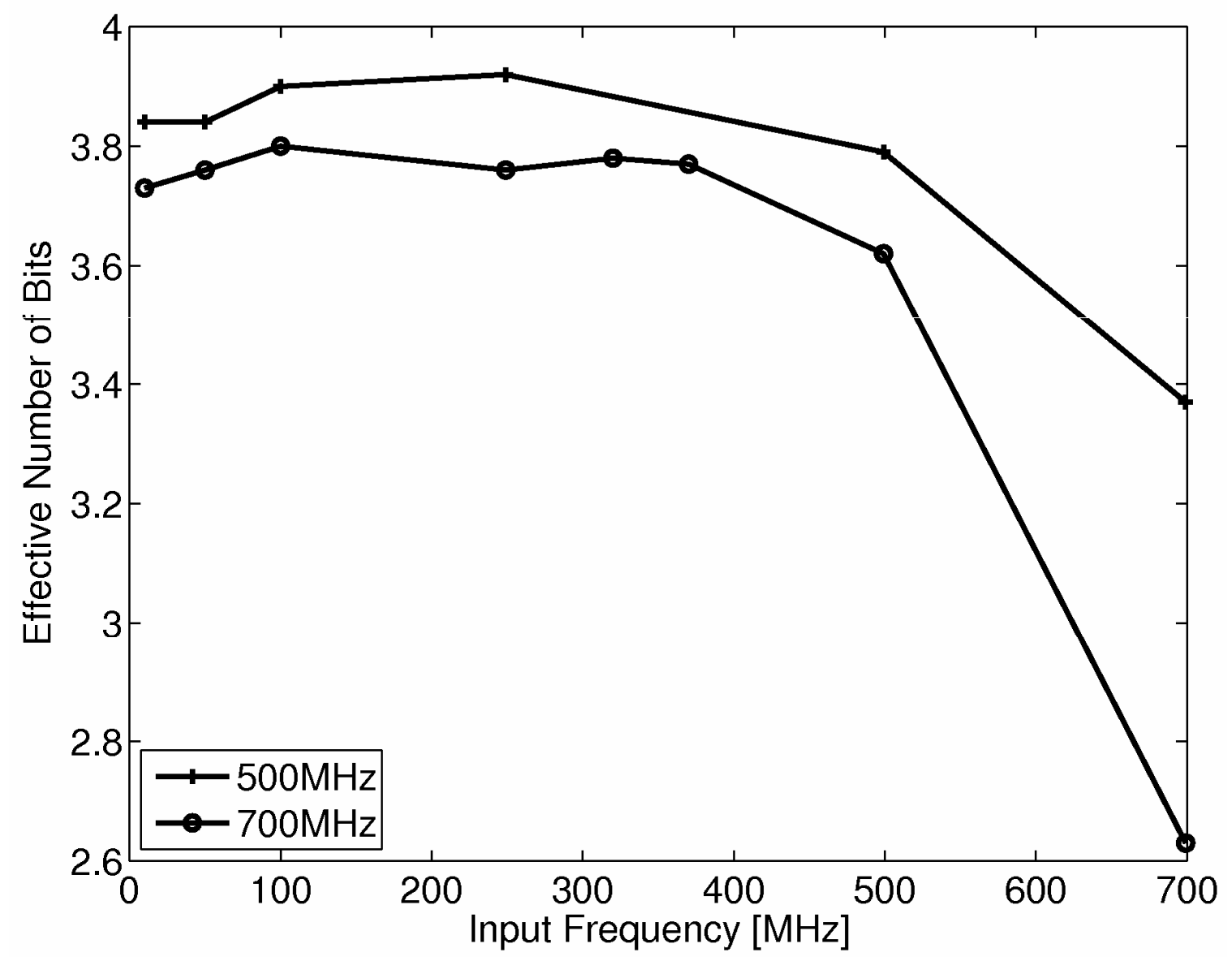


Table 1.

\begin{tabular}{|c|c|c|c|c|c|c|c|c|}
\hline & $\begin{array}{c}\text { Technol } \\
\text { ogy }\end{array}$ & \begin{tabular}{|c|}
$\#$ \\
of \\
bit \\
$s$
\end{tabular} & DNL & INL & $\begin{array}{c}\text { ENO } \\
\text { B @ } \\
\text { Nyqu } \\
\text { ist }\end{array}$ & $\begin{array}{c}\text { Sampl } \\
\text { e } \\
\text { Frequ } \\
\text { ency } \\
(\mathrm{MHz})\end{array}$ & $\begin{array}{l}\text { Pow } \\
\text { er } \\
(\mathrm{m} \\
\text { W) }\end{array}$ & $\begin{array}{c}\text { Fo } \\
M \\
\text { (pj/c } \\
\text { onv) }\end{array}$ \\
\hline This work & $0.18 \mu \mathrm{m}$ & 4 & $<0.19$ & $<0.27$ & 3.77 & 700 & 4.43 & 0.46 \\
\hline [2] & $0.18 \mu \mathrm{m}$ & 4 & $<0.25$ & $<0.17$ & $\begin{array}{l}3.8 \\
3.7\end{array}$ & $\begin{array}{l}500 \\
1000\end{array}$ & $\begin{array}{l}5.2 \\
10.6\end{array}$ & $\begin{array}{l}0.73 \\
0.81\end{array}$ \\
\hline [4] & $0.18 \mu \mathrm{m}$ & 4 & 0.4 & 1.1 & 3.24 & 400 & 20 & 5.30 \\
\hline [5] & $0.18 \mu \mathrm{m}$ & 4 & 0.04 & 0.06 & 3.61 & 2000 & 42 & 1.72 \\
\hline [6] & $0.13 \mu \mathrm{m}$ & 6 & 0.49 & 0.42 & 4.69 & 1600 & 180 & 4.36 \\
\hline [7] & $0.13 \mu \mathrm{m}$ & 6 & $<0.6$ & $<0.4$ & $\begin{array}{l}5.6 \\
5.7\end{array}$ & $\begin{array}{l}600 \\
1200\end{array}$ & $\begin{array}{l}90 \\
160\end{array}$ & $\begin{array}{l}3.09 \\
2.56\end{array}$ \\
\hline [8] & $0.13 \mu \mathrm{m}$ & 5 & $<0.24$ & $<0.39$ & $\begin{array}{l}4.44 \\
4.54\end{array}$ & $\begin{array}{l}2000 \\
3200\end{array}$ & $\begin{array}{l}120 \\
120\end{array}$ & $\begin{array}{l}3.07 \\
4.30\end{array}$ \\
\hline
\end{tabular}

